Corrigendum

\title{
Corrigendum to "Generating Point Cloud from Measurements and Shapes Based on Convolutional Neural Network: An Application for Building 3D Human Model"
}

\author{
Mau Tung Nguyen, ${ }^{1,2}$ Thanh Vu Dang $\mathbb{D}^{3},{ }^{3}$ Minh Kieu Tran Thi, ${ }^{1}$ and Pham The Bao $\mathbb{D}^{3}$ \\ ${ }^{1}$ Hanoi University of Science and Technology, School of Textile-Leather and Fashion, Hanoi, Vietnam \\ ${ }^{2}$ Industrial University of Ho Chi Minh City, Ho Chi Minh City, Vietnam \\ ${ }^{3}$ Sai Gon University, Ho Chi Minh City, Vietnam \\ Correspondence should be addressed to Pham The Bao; ptbao2005@gmail.com
}

Received 17 October 2019; Accepted 12 November 2019; Published 28 June 2020

Copyright (c) 2020 Mau Tung Nguyen et al. This is an open access article distributed under the Creative Commons Attribution License, which permits unrestricted use, distribution, and reproduction in any medium, provided the original work is properly cited.

In the article titled "Generating Point Cloud from Measurements and Shapes Based on Convolutional Neural Network: An Application for Building 3D Human Model" [1], there was an error in affiliation number 1 . In the corrected affiliation, "Hanoi" has been added to the institution name, and the city has been corrected from "Ho Chi Minh City" to "Hanoi." The correct affiliation appears below.

Hanoi University of Science and Technology, School of Textile-Leather and Fashion, Hanoi, Vietnam.

\section{References}

[1] M. T. Nguyen, T. V. Dang, M. K. Tran Thi, and P. T. Bao, "Generating point cloud from measurements and shapes based on convolutional neural network: an application for building 3D human model," Computational Intelligence and Neuroscience, vol. 2019, Article ID 1353601, 15 pages, 2019. 